\title{
Phase II trial of combined treatment consisting of preoperative S-1 plus cisplatin followed by gastrectomy and postoperative S-1 for stage IV gastric cancer
}

\author{
Seiji Satoh $\cdot$ Hiroshi Okabe $\cdot$ Satoshi Teramukai $\cdot$ Suguru Hasegawa $\cdot$ \\ Nobuhiro Ozaki $\cdot$ Shugo Ueda $\cdot$ Ayumi Tsuji $\cdot$ Satomi Sakabayashi • \\ Masanori Fukushima • Yoshiharu Sakai
}

Received: 28 March 2011 / Accepted: 17 May 2011/Published online: 11 June 2011

(c) The International Gastric Cancer Association and The Japanese Gastric Cancer Association 2011

\begin{abstract}
Background To improve the poor prognosis in patients with stage IV (StIV) gastric cancer (GC), we conducted a multicenter phase II study of preoperative S-1 plus cisplatin followed by gastrectomy and postoperative S-1 for StIV GC (the protocol is registered at the clinical trial site of the National Cancer Institute; KYUH-UHA-GC03-01, NCT00088816).

Methods Eligibility criteria included histologically proven StIVGC. Patients received S-1 $\left(80 \mathrm{mg} / \mathrm{m}^{2} /\right.$ day, days $1-21)$ plus cisplatin $\left(60 \mathrm{mg} / \mathrm{m}^{2}\right.$ on day 8$)$ for 2 courses. After preoperative chemotherapy (CTx), radical gastrectomy was performed. Postoperative $\mathrm{S}-1\left(80 \mathrm{mg} / \mathrm{m}^{2} /\right.$ day, days 1-14) was administered every 3 weeks for 1 year.

Results Fifty-one patients were enrolled and all patients were followed for more than 2 years. The 2-year overall survival and progression-free survival rates were $43.1 \%$ (95\% confidence interval [CI] 29.4-56.1\%) and 33.3\% (95\% CI 20.9-46.2\%), respectively. Preoperative chemotherapy was accomplished in 44 patients $(86.3 \%)$. These 44
\end{abstract}

S. Satoh $\cdot$ H. Okabe $\cdot$ S. Hasegawa $\cdot$ N. Ozaki $\cdot$ S. Ueda

Y. Sakai

Kyoto University Surgical Oncology Group, Kyoto, Japan

A. Tsuji · S. Sakabayashi · M. Fukushima

Translational Research Informatics Center, Kobe, Japan

S. Teramukai

Translational Research Center, Kyoto University Hospital,

Kyoto, Japan

S. Satoh $(\square)$

Department of Upper Gastrointestinal Surgery,

Fujita Health University, 1-98 Dengakugakubo,

Kutsukake-cho, Toyoake, Aichi, Japan

e-mail: ssatoh415@gmail.com patients underwent surgery and R0 resection was achieved in 26. The rate of R0 resection for GC with a single StIV factor $(n=24)$ was $79.2 \%$ and that for GC with multiple StIV factors $(n=27)$ was $25.9 \%$. All patients with cancer cells in peritoneal washings (cytology $[\mathrm{Cy}] 1$ ) alone $(n=12)$ became Cy0 after preoperative chemotherapy. Postoperative chemotherapy was completed in 11 patients, including 8 with Cy1 alone. No treatment-related death was recorded. Recurrences were observed in 14 patients after $\mathrm{R} 0$ resection. The most frequent recurrence site was the peritoneum. Patients who underwent R0 resection and those with Cy1 alone had a better survival.

Conclusions This perioperative treatment was safe and feasible for StIVGC but failed to show a survival benefit. In patients with StIVGC with $\mathrm{Cy} 1$ alone this treatment resulted in a better prognosis.

Keywords Gastric cancer - Induction chemotherapy · Surgery $\cdot$ S-1 plus cisplatin

\section{Introduction}

Gastric cancer (GC) is one of the leading causes of cancer death worldwide [1]. It is often diagnosed at an advanced stage. The prognosis of stage IV GC is poor (e.g., the 1-, 2-, and 5-year survival rates of stage IV GC are approximately 40,20 , and $<10 \%$, respectively $[2,3]$ ), even if patients are treated surgically.

Recently, several novel regimens of combined chemotherapy, including S-1 plus cisplatin, have produced improved overall survival (OS) rates in patients with unresectable stage IV GC [4-6]. The median survival time in patients treated with S-1 plus cisplatin was 13.0 months, and the response rate with this regimen was $54 \%$. But its 
2-year OS rate, $23.6 \%$ (95\% confidence interval [CI] 16.8-30.4), was still dismal [6].

Combinations of some highly effective chemotherapeutic regimens and surgery have given favorable results in resectable, locally advanced GC [7-11]. Preoperative chemotherapy for advanced GC possesses theoretical benefits. First, this chemotherapy usually shrinks cancerous tissue, increasing the likelihood of R0 resection with extended surgery. Second, more intensive chemotherapy than that used postoperatively is possible, with a higher compliance rate. Third, distant occult metastases can be treated before local therapy has begun. Furthermore, postoperative $\mathrm{S}-1$ alone has been proven beneficial for treating stage II and stage III GC [12]. Hence, one of the most potentially favorable multimodal treatments for stage IV GC would be a combination of preoperative administration of S-1 plus cisplatin, subsequent gastrectomy with D2 nodal dissection, and postoperative S-1 administration. The present study was conducted to evaluate the efficacy, feasibility, and safety of this novel multimodal treatment for stage IV GC.

\section{Patients and methods}

This was a prospective multicenter phase II study conducted at eight centers of the Kyoto University Surgical Oncology Group in Japan between May 2003 and March 2008. This protocol is registered at the clinical trial site of the National Cancer Institute (KYUH-UHA-GC03-01, NCT00088816).

\section{Patients}

Eligibility criteria included: (1) pretreatment (pre-) histologically proven stage IV GC (pre-N3, pre-T4N2, pre-H1, pre-P1, pre-Cy1, pre-M1) diagnosed by pretreatment helical computed tomography (CT) and staging laparoscopy within 4 weeks before registration, (2) no prior therapy, (3) Eastern Clinical Oncology Group (ECOG) performance status of 0 or 1 , and (4) age of $20-80$ years. Patients must have had no signs of organ failure, including that of bone marrow, heart, lungs, and kidneys. Acceptable hematologic (white blood cell count $\geq 4,000 / \mu \mathrm{L}$ and $\leq 12,000 / \mu \mathrm{L}$, neutrophil count $\geq 2,000 / \mu \mathrm{L}$, platelet count $\geq 100,000 / \mu \mathrm{L}$, hemoglobin $\geq 9.0 \mathrm{~g} / \mu \mathrm{L}$ ), renal (creatinine clearance $\geq$ $50 \mathrm{~mL} / \mathrm{min}$ ), hepatic (aspartate aminotransferase and alanine aminotransferase $\leq 2.5$ times the upper limit at each institution, serum total bilirubin $\leq 1.5 \mathrm{mg} / \mathrm{dL}$ ), and respiratory function (arterial partial pressure of oxygen $\left[\mathrm{PaO}_{2}\right] \geq 70$ torr in room air) was required for enrollment in the study.
Exclusion criteria included active gastrointestinal bleeding, infection, diarrhea, simultaneous double cancer, pregnancy, interstitial pneumonia, bowel obstruction, past history of myocardial infarction, massive ascites, severe drug allergy, or severe diabetes. The protocol was approved by the ethics review committees of all participating centers, and all patients gave written informed consent. All patients were registered centrally at the Translational Research Informatics Center, Kobe, Japan, where data management and statistical analysis were performed.

\section{Preoperative chemotherapy}

Preoperative chemotherapy was administered for two cycles. Each cycle consisted of daily oral administration of S-1 for 21 days $\left(80 \mathrm{mg} / \mathrm{m}^{2} /\right.$ day, days $\left.1-21\right)$ and intravenous cisplatin $\left(60 \mathrm{mg} / \mathrm{m}^{2}\right)$, with hydration, on day 8; followed by a 2 -week recovery period [6]. The toxicity of the induction chemotherapy was assessed on the basis of the National Cancer Institute's common toxicity criteria version 2.0 (NCI-CTC) [13]. If patients had grade 3/4 hematologic or nonhematologic toxicity, including grade 2 or above diarrhea, liver dysfunction, stomatitis, and other toxicities of grade 3 or above, chemotherapy was postponed until recovery. If recovery did not occur within 4 weeks, the chemotherapy was stopped.

Some 7-13 days after each course, resectability and clinical response were evaluated mainly on the basis of CT findings. If curative resection was possible after the second course, the patient underwent surgery 2-4 weeks after completion of the chemotherapy. If patients experienced disease progression or severe adverse events, the protocol was discontinued. Treatment after the discontinuation of preoperative chemotherapy was at the physician's discretion.

\section{Surgery}

The surgical criteria included possibility of R0 resection, no evidence of infection, no signs of organ failure, and a neutrophil count of more than $1,500 / \mu \mathrm{L}$. After laparotomy, resectability was again evaluated. The standard surgical procedure was gastrectomy with $\mathrm{D} 2$ nodal dissection. The extent of gastrectomy (total or subtotal) depended on the site and size of the primary tumor. For R0 resection, paraaortic nodal dissection (D3), splenectomy and/or distal pancreatectomy, or partial hepatectomy was attempted if cytologic findings were negative. Palliative gastrectomy was attempted in noncurative cases if the tumor was symptomatic and resectable. 
Postoperative chemotherapy

Postoperative S-1 (80 mg/m²/day, days 1-14) was administered every 3 weeks for 1 year. Eligibility criteria for postoperative chemotherapy included resection of the primary lesion and no definite disease progression at surgery. If gastrectomy was not curative, cessation of the protocol was at the physicians' discretion. Other eligibility criteria were the same as those for preoperative chemotherapy. For 1 year, S-1 was repeatedly administered in a 2-week cycle, followed by 1 week of rest. Criteria for discontinuation and cessation of the protocol were the same as those for preoperative chemotherapy. If recurrence or progressive disease was confirmed, the protocol was stopped. After completion of the protocol without recurrence, no further treatment was administered until tumor recurrence was identified. Treatment for recurrence or progressive disease was at the physicians' discretion.

\section{Endpoints and evaluation}

The primary endpoint was the 2-year OS rate, because the prognosis of stage IV GC is poor. The secondary endpoints were the progression-free survival (PFS) rate, objective response rate, pathologic response rate, $\mathrm{R} 0$ resection rate, surgical complications, the first recurrence sites, and toxic reactions. R0 resection was defined as pathologically confirmed complete resection of the cancerous lesions with negative peritoneal washing cytology.

The pretreatment stage at registration (pre-stage) was diagnosed according to the Japanese Gastric Cancer Association (JGCA) staging system (13th edition) [14] on the basis of CT and staging laparoscopic findings. If the short-axis diameter of the lymph nodes was greater than $10 \mathrm{~mm}$ on a CT scan, the lymph nodes were diagnosed as "metastatic" $[15,16]$. The anatomic location of metastatic lymph nodes was also identified on CT scans. According to the JGCA staging system, $\mathrm{N}$ stage was defined by the anatomic location of the metastatic lymph nodes. At staging laparoscopy, $\mathrm{T}$ stage was diagnosed as pre-T3 (tumor penetration of serosa; SE) if the tumor was definitely exposed on the serosal surface. If cancerous changes on the serosal surface were considered minimal at staging laparoscopy, the $\mathrm{T}$ stage was diagnosed as pre-T2 (tumor invasion of muscularis propria; MP, tumor invasion of subserosa; SS). The diagnosis of pre-T4 (tumor invasion of adjacent structures; SI) was assessed by CT and laparoscopic findings. Cytology and peritoneal tumor dissemination were also assessed at pretreatment staging laparoscopy [17-20] and at surgery. The cells collected by peritoneal washing were examined cytopathologically using conventional Papanicolaou and Giemsa staining.
When definite cancer cells or clusters were identified, patients were diagnosed as pre-Cy1, according to the Japanese classification of gastric carcinoma of the JGCA [14]. Objective tumor response was evaluated using the Response Evaluation Criteria in Solid Tumors (RECIST) version 1.0 on the basis of the CT findings [21]. Postoperative final tumor status (post-stage) was diagnosed by comprehensive findings based on clinical, surgical, and pathological findings according to the JGCA classification [14]. Surgical specimens were evaluated pathologically and graded according to the proportion of tumor affected by degeneration or necrosis [11]: grade 0 , no part of tumor affected; grade 1a, less than one-third affected; grade $1 \mathrm{~b}$, between one-third and two-thirds affected; grade 2, between two-thirds and the entire tumor affected; and grade 3 , no residual tumor. The toxicity of preoperative and postoperative chemotherapy was assessed on the basis of NCI-CTC version 2, as described earlier.

Statistical analyses

All data from eligible patients were based on the intentionto-treat principle. This study was designed as a phase II trial with a single stage of accrual. The threshold 2-year survival probability was estimated to be $35 \%$, determined by examining historical data from the Department of Gastroenterological Surgery, Kyoto University, that were collected from 1996 to 2002 ( $n=43)$. The expected 2-year survival rate for this therapy was estimated to be $55 \%$. Assuming that two-sided type I errors were 5\% and that the power was $80 \%$, the required sample size was calculated to be 46 . The planned sample size was set at 50 , with the consideration of approximately $5 \%$ of patients being ineligible. OS and PFS were estimated using the Kaplan-Meier

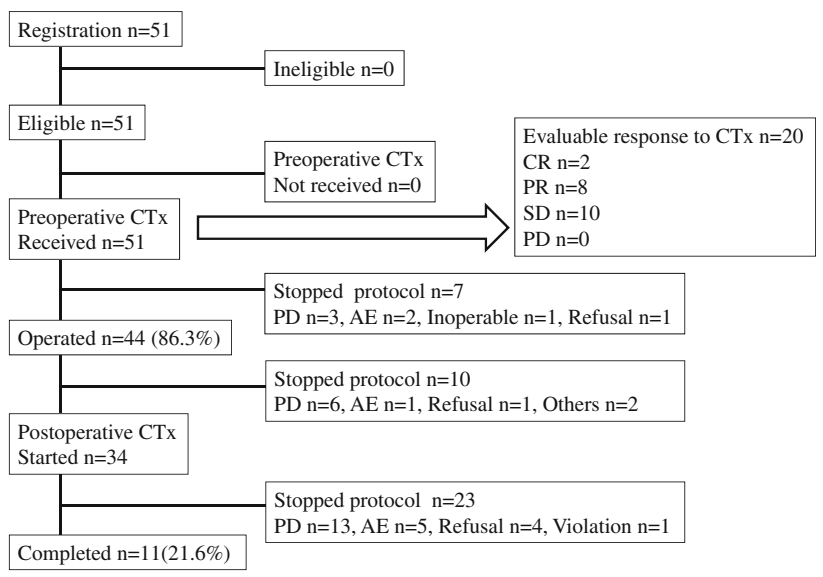

Fig. 1 Flow diagram of 51 eligible patients. CTx chemotherapy, $C R$ complete response, $P R$ partial response, $S D$ stable disease, $P D$ progressive disease, $A E$ adverse effects 
Table 1 Characteristics of patients and tumor status before and after treatment

\begin{tabular}{lll}
\hline & $n$ & $\%$ \\
\hline $\begin{array}{l}\text { Age, years, median (range) } \\
\text { Gender }\end{array}$ & $63(35-79)$ & \\
$\quad$ Male & 29 & 56.9 \\
Female & 22 & 43.1 \\
ECOG performance status & & \\
0 & 44 & 86.3 \\
1 & 7 & 13.7 \\
Histology & & 25.5 \\
Intestinal & 13 & 74.5 \\
Diffuse & 38 & Post- $(n=44)$ \\
\hline & Pre- $(n=51)$ & \\
\hline
\end{tabular}

Stage

IA

IB

II

IIIA

IIIB

IV

T stage

T1 (M, SM)

T2 (MP, SS)

T3 (SE)

T4 (SI)

TX

$\mathrm{N}$ stage

No

$\mathrm{N} 1$

$\mathrm{N} 2$

N3

NX

Peritoneal cytology

Cy0

Cy1

CyX

Peritoneal metastasis

P0

P1

Liver metastasis

Ho

H1

Distant metastasis

\begin{tabular}{lrr} 
M0 & 46 & 43 \\
M1 & 5 & 1 \\
Pathological response (primary lesion) & \\
Grade 0 & 6 \\
Grade 1a & 15 \\
Grade 1b & 6 \\
\hline
\end{tabular}

Table 1 continued

\begin{tabular}{|c|c|c|}
\hline & Pre- $(n=51)$ & Post- $(n=44)$ \\
\hline Grade 2 & & 14 \\
\hline Grade 3 & & 0 \\
\hline Not resected & & 3 \\
\hline
\end{tabular}

ECOG Eastern Cooperative Oncology Group, Pre- pretreatment tumor status at registration, Post-postoperative final tumor status diagnosed by comprehensive findings based on clinical, surgical and pathological findings according to the Japanese Gastric Cancer Association (JGCA) classification [14], $M$ tumor invasion of mucosa and/or muscularis mucosa, $S M$ tumor invasion of submucosa, $M P$ tumor invasion of muscularis propria, $S S$ tumor invasion of subserosa, $S E$ tumor penetration of serosa, SI tumor invasion of adjacent structures

method. All analyses were performed using SAS version 9.1 (SAS Institute, Cary, NC, USA).

\section{Results}

Patient characteristics

Between May 2003 and March 2008, 51 patients with stage IV GC were enrolled in this study and underwent preoperative chemotherapy. Disposition of the enrolled patients is shown in Fig. 1. No patients were ineligible, and all were observed for more than 2 years after registration. Table 1 shows the characteristics and tumor status of the 51 eligible patients. The median follow-up period of all patients was 19.2 months (range 4.5-81.5). Patients with a single prestage IV factor $(n=24)$ included 12 cases of pre-Cy1, 5 cases of pre-P1, 5 cases of pre-N3, and 2 cases of pre-H1. Simultaneous multiple pre-stage IV factors were observed in the remaining 27 patients. Patients with multiple prestage IV factors $(n=27)$ included 1 case of pre-(Cy1, P1, N3, M1), 1 case of pre-(Cy1, P1, N3), 2 cases of pre-(Cy1, P1, T4N2), 1 case of pre-(Cy1, P1, H1), 1 case of pre-(Cy1, H1, M1), 15 cases of pre-(Cy1, P1), 1 case of pre-(Cy1, N3), 1 case of pre-(Cy1, T4N2), 1 case of pre-(Cy1, H1), and 3 cases of pre-(N3, M1).

Survival

The 2-year OS and PFS rates were $43.1 \%$ (95\% CI 29.4-56.1) and $33.3 \%$ (95\% CI 20.9-46.2), respectively (Fig. 2a, b). The median OS and PFS were 19.2 and 9.3 months, respectively. There were significant differences in OS in favor of both GC with R0 resection $(p<0.0001)$ and GC with pre-Cy1 alone without any other stage IV factors $(p=0.0041)$, as shown in Fig. 2c, d. The 2-year OS of GC with pre-Cy1 alone was $75.0 \%$ (95\% CI 40.8-91.2) and that of others was 33.3\% (95\% CI 19.3-48.0). 

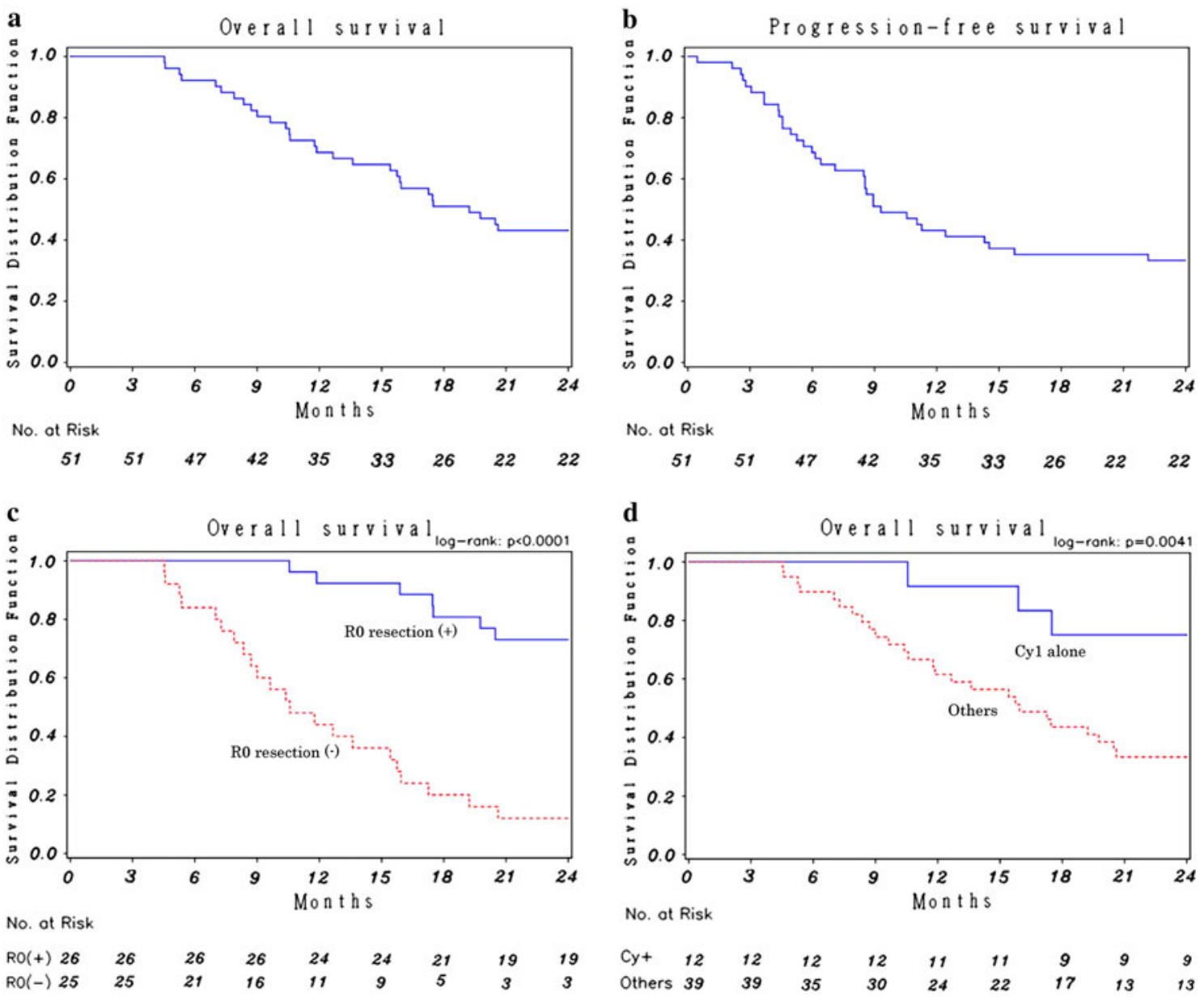

Fig. 2 Kaplan-Meier survival curves for the 51 eligible patients. a Overall survival, b progression-free survival. c Overall survival in patients grouped according to R0 resection or not. d Overall survival in patients grouped according to pretreatment $\mathrm{Cy} 1$ alone and others

Objective response to preoperative chemotherapy and adverse events

Measurable lesions were confirmed in 20 patients. The objective response rate for these lesions, according to the RECIST, was $50.0 \%$ (95\% CI 27.2-72.8). Preoperative chemotherapy was completed in 44 patients $(86.3 \%)$. Reasons for stopping treatment are noted in Fig. 1.

Toxic reactions of grade 3 or above are listed in Table 2. Severe adverse events occurring during preoperative chemotherapy were noted in two patients $(3.9 \%)$. Grade 4 toxic reactions of leukocytopenia, neutropenia, and anemia were observed in one patient. Cardiopulmonary arrest after diarrhea was observed in another patient, who was resuscitated successfully.

\section{Surgical outcome}

Surgery was performed in 44 patients, including total gastrectomy in 30 , distal gastrectomy in 11 , simple laparotomy in two, and gastrojejunostomy in one patient. The median (range) blood loss and surgery duration were $669(0-3,228) \mathrm{mL}$ and 286 (50-447) min, respectively. Extended nodal dissection was performed in 36 patients (D2, 32; D3, 4). Palliative gastrectomy with D0/D1 was performed in 5 patients to maintain oral intake. Combined resection was performed in 35 patients, including additional resection of 62 organs; namely, the spleen $(n=25)$, gallbladder $(n=21)$, distal pancreas $(n=7)$, colon $(n=3)$, liver $(n=2)$, mesocolon $(n=2)$, diaphragm $(n=1)$, and adrenal gland $(n=1)$. The surgical complication rate was $18.2 \%$, including three cases of pancreatic fistula and abdominal abscess, and one case of anastomotic leakage.

The postoperative final tumor status (post-stage) of the 44 patients who underwent surgery was determined with comprehensive findings based on clinical, surgical, and pathologic findings according to the JGCA classification (Table 1).

$\mathrm{R} 0$ resection rate

According to the pretreatment evaluation, 31 patients were diagnosed as "unresectable". After induction chemotherapy, 44 patients were diagnosed as "resectable" by CT and 
Table 2 Adverse events of chemotherapy according to NCI-CTC version 2

NCI-CTC National Cancer Institute common toxicity criteria, $C D D P$ cisplatin, $D I C$ disseminated intravascular coagulation

Table 3 Relationship between pre-stage IV factors and R0 resection

Pre-pretreatment clinical tumor status at registration, $M S T$ median survival time, $C I$ confidence interval, $N E$ not estimated

\begin{tabular}{|c|c|c|c|c|}
\hline & \multicolumn{2}{|c|}{ Preoperative S- $1+$ CDDP $(n=51)$} & \multicolumn{2}{|c|}{ Postoperative S-1 $(n=34)$} \\
\hline & Grade $3 / 4$ & $\%$ & Grade 3/4 & $\%$ \\
\hline Leukocytopenia & 6 & 12 & 1 & 3 \\
\hline Neutropenia & 13 & 25 & 4 & 12 \\
\hline Thrombocytopenia & 2 & 4 & 0 & 0 \\
\hline Anemia & 6 & 12 & 1 & 3 \\
\hline Fatigue & 5 & 10 & 2 & 6 \\
\hline Anorexia & 11 & 22 & 3 & 9 \\
\hline Nausea & 6 & 12 & 2 & 6 \\
\hline Vomiting & 4 & 8 & 0 & 0 \\
\hline Diarrhea & 1 & 2 & 2 & 6 \\
\hline DIC & 1 & 2 & 0 & 0 \\
\hline Dehydration & 1 & 2 & 0 & 0 \\
\hline Dizziness & 0 & 0 & 1 & 3 \\
\hline
\end{tabular}

\begin{tabular}{lcccc}
\hline & No. & R0 & $\%$ R0 & MST (95\% CI) (months) \\
\hline Total & 51 & 26 & 51.0 & $19.2(15.4-43.5)$ \\
Multiple pre-stage IV factors & 27 & 7 & 25.9 & $11.9(9.0-17.5)$ \\
Single pre-stage IV factor & 24 & 19 & 79.2 & $56.3(19.8-\mathrm{NE})$ \\
Pre-Cy1 & 12 & 12 & 100 & NE (56.3-NE) \\
Pre-P1 & 5 & 2 & 40 & $43.5(7.9-\mathrm{NE})$ \\
Pre-N3 & 5 & 4 & 80 & $31.6(17.3-\mathrm{NE})$ \\
Pre-H1 & 2 & 1 & 50 & NE (15.9-NE) \\
\hline
\end{tabular}

they underwent surgery. R0 resection after induction chemotherapy was accomplished in 26 patients. Noncurative factors in $\mathrm{R} 1 / 2$ cases $(n=18)$ included 3 cases of unresectable primary tumor, 13 cases of post-P1 and/ or Cy1, 1 case of residual nodal metastasis, and 1 case of multiple liver metastasis. The relationship between stage IV status at registration (pre-stage IV) and R0 resection is summarized in Table 3. Twelve patients showed preCy1 without other c-stage IV factors (Cy1 alone). In these 12 patients, eradication of free peritoneal gastric cancer cells (FPGCs) with preoperative chemotherapy was observed and this led to R0 resection. Of 15 patients with pre-Cy0, two patients became post-Cy1 after preoperative chemotherapy.

\section{Postoperative chemotherapy and recurrence}

Of the 26 patients that underwent R0 resection, three patients showed no recurrence without postoperative chemotherapy. Reasons for not performing postoperative chemotherapy in these three patients were that 1 patient was ineligible for the starting criteria of the postoperative chemotherapy, 1 patient had prolonged pneumonia, and 1 patient refused. Postoperative chemotherapy was started in 34 patients, including 23 patients with $\mathrm{R} 0$ resection, and
Table 4 Postoperative chemotherapy and recurrence in patients with R0 resection $(n=23)$

\begin{tabular}{lrrl}
\hline & Start & Completion & Rec.-free $(\%)$ \\
\hline Total & 23 & 11 & $9(39)$ \\
Multiple pre-stage IV factors & 7 & 2 & $0(0)$ \\
Single pre-stage IV factor & 16 & 9 & $9(56)$ \\
Pre-Cy1 & 11 & 8 & 7 \\
Pre-P1 & 1 & 0 & 0 \\
Pre-N3 & 3 & 0 & 1 \\
Pre-H1 & 1 & 1 & 1 \\
\hline
\end{tabular}

Pre- pretreatment clinical tumor status at registration, Rec.-free recurrence free

was completed in 11 . No patients with noncurative resection completed 1 year of postoperative chemotherapy $(n=11)$. The relationship between postoperative chemotherapy and recurrence in R0 resection patients $(n=23)$ is summarized in Table 4 . The adverse effects of the postoperative chemotherapy are presented in Table 2. Grade 4 toxic effects of neutropenia, fatigue, and dizziness were observed in one patient. Throughout the treatment period, severe adverse events were recorded in three patients (5.9\%). No treatment-related death occurred. Of the 26 patients with R0 resection, 14 experienced recurrence. The 
most frequent recurrence site was the peritoneum $(n=8)$, followed by the lymph nodes $(n=3)$.

\section{Discussion}

The results of the present study indicate that the multimodal treatment used was safe and well tolerated by patients with stage IV GC. The clinical response rate for the preoperative chemotherapy was compatible with that reported in a previous study [6]. Surgical morbidity was acceptable. No treatment-related death occurred. With a mature follow-up, the 2-year OS rate of this treatment was $43 \%$ (95\% CI 29.4-56.1). This appeared to be better than the 2-year OS of 20-25\% that was estimated from previous studies of stage IV GC patients treated with chemotherapy alone or with surgery and postoperative chemotherapy [2, $3,6,22]$. However, our study failed to show a survival benefit, because our threshold 2-year survival probability was estimated to be $35 \%$.

Although most of the eligible patients in our study had microscopic or macroscopic peritoneal dissemination, R0 resection was achieved in $51 \%$ of the patients after preoperative chemotherapy with S-1 plus cisplatin. R0 resection is reported to be one of the most reliable prognostic indicators for patients after preoperative chemotherapy [10, 17]. In line with these reports, the $\mathrm{R} 0$ resection group in the present study exhibited a significantly better prognosis. It is well known that the prognosis in patients with GC with multiple stage IV factors is miserable [23-25]. By virtue of staging laparoscopy, we frequently confirmed overt peritoneal carcinomatosis (pre-P1) or FPGCs (pre-Cy1). Other synchronous pre-stage IV factors were identified in $80 \%$ of pre-P1 patients and $66 \%$ of pre-Cy1 patients. The R0 resection rate was low in these patients, mainly due to remaining peritoneal carcinomatosis or FPGCs. Preoperative $\mathrm{S}-1$ plus cisplatin might be inadequate for treating GC with multiple c-stage IV factors. In this situation, preoperative staging laparoscopy after the induction chemotherapy could have been useful to prevent futile laparotomy.

As for GC with a single pre-stage IV factor, the R0 resection rate in our study was as high as $79 \%$. R0 resection due to eradication of FPGCs with preoperative chemotherapy was recorded in all patients with pre-Cy1 alone, leading to an improved prognosis. Generally, the 2-year overall survival (OS) rate of GC with pre-Cy1 alone (around 25-30\%) is poor, even when conventional postoperative chemotherapy is administered [22, 26, 27]. In general, preoperative chemotherapy using a conventional regimen is insufficient for GC with pre-Cy1 [28]. Recently, improved prognosis was reported in patients with pre-Cy1 alone as a result of the eradication of FPGCs with preoperative cisplatin and folic acid plus fluorouracil. However, the eradication rate was low (37\%), and a shift from negative to positive cytology findings during preoperative chemotherapy was observed in $24 \%$ of patients [29]. As for adjuvant chemotherapy for GC with post-Cy1 alone, the 2-year OS rate of these patients who underwent radical surgery followed by $\mathrm{S}-1$ alone was reported to be $47 \%$ [30]. In contrast, in the present study, the eradication rate of FPGCs was $100 \%$, leading to a 2-year OS rate of $75.0 \%$ (95\% CI 40.8-91.2) in the patients with pre-Cy1 alone, and two patients $(3.9 \%)$ had a shift from negative to positive cytology findings. This result was compatible with findings in our preliminary reports $[7,31]$. S-1 shows a high rate of transfer into the peritoneal cavity [32]. Compared with S-1 alone, S-1 plus cisplatin improves the prognosis of patients with peritoneal metastasis [6]. These features of S-1 plus cisplatin might have contributed to the high eradication rate of FPGCs in the present study. Also, due to the high eradication rate of FPGCs, pretreatment staging laparoscopy is strongly recommended to distinguish advanced GC with pre-Cy1 from that with pre-Cy0.

Low compliance is often reported for postoperative chemotherapy, because the toxicity of combination regimens cannot be tolerated by patients after gastrectomy $[8$, 9]. Postoperative S-1 plus cisplatin has also been reported to be too toxic after gastrectomy [33]. To improve compliance with postoperative chemotherapy, S-1 alone was adopted as a postoperative regimen [12]. Consequently, the toxic profile of postoperative $\mathrm{S}-1$ alone was milder than that of preoperative S-1 plus cisplatin. In the present study, the low completion rate of the postoperative chemotherapy was due to tumor progression in patients with multiple stage IV factors. Despite the postoperative chemotherapy, all patients with multiple pre-stage IV factors experienced recurrence after R0 resection. On the other hand, the recurrence-free rate of patients with a single pre-stage IV factor was $56 \%$ after postoperative chemotherapy. In particular, due to the low frequency of recurrence, patients with pre-Cy1 alone showed a high postoperative chemotherapy completion rate. Postoperative chemotherapy with S-1 alone might be effective in treating stage IV GC if the latent tumor burden is minimal after $\mathrm{R} 0$ resection.

In conclusion, the administration of preoperative S-1 plus cisplatin, followed by surgery and postoperative S-1, is safe and feasible for stage IV GC. However, this multimodal approach failed to show a beneficial effect for GC with multiple stage IV factors. Although the sample size in our study was small and 2 of 15 pre-Cy0 patients became post-Cy1, it seems that patients with pre-Cy1 with no additional non-curative factors might be good candidates for the present approach. Further clinical investigations for this subset should be undertaken using promising perioperative chemotherapy. 
Acknowledgments This study was supported by the Foundation for Biomedical and Innovation, Japan.

Conflict of interest The authors indicated no potential conflicts of interest.

\section{References}

1. Parkin DM, Bray FI, Devesa SS. Cancer burden in the year 2000. The global picture. Eur J Cancer. 2001;37(Suppl 8):S4-66.

2. Japanese Gastric Cancer Association Registration Committee, Maruyama K, Kaminishi M, Hayashi K, Isobe Y, Honda I, et al. Gastric cancer treated in 1991 in Japan: data analysis of nationwide registry. Gastric Cancer. 2006;9:51-66.

3. Nio Y, Tsubono M, Kawabata K, Masai Y, Hayashi H, Meyer C, et al. Comparison of survival curves of gastric cancer patients after surgery according to the UICC stage classification and the General Rules for Gastric Cancer Study by the Japanese Research Society for gastric cancer. Ann Surg. 1993;218:47-53.

4. Van Cutsem E, Moiseyenko VM, Tjulandin S, Majlis A, Constenla M, Boni C, et al. V325 Study Group. Phase III study of docetaxel and cisplatin plus fluorouracil compared with cisplatin and fluorouracil as first-line therapy for advanced gastric cancer: a report of the V325 Study Group. J Clin Oncol. 2006;24:4991-7.

5. Ross P, Nicolson M, Cunningham D, Valle J, Seymour M, Harper $\mathrm{P}$, et al. Prospective randomized trial comparing mitomycin, cisplatin, and protracted venous-infusion fluorouracil (PVI 5-FU) With epirubicin, cisplatin, and PVI 5-FU in advanced esophagogastric cancer. J Clin Oncol. 2002;20:1996-2004.

6. Koizumi W, Narahara H, Hara T, Takagane A, Akiya T, Takagi M, et al. S-1 plus cisplatin versus S-1 alone for first-line treatment of advanced gastric cancer (SPIRITS trial): a phase III trial. Lancet Oncol. 2008;9:215-21.

7. Okabe H, Ueda S, Obama K, Hosogi H, Sakai Y. Induction chemotherapy with S-1 plus cisplatin followed by surgery for treatment of gastric cancer with peritoneal dissemination. Ann Surg Oncol. 2009;16:3227-36.

8. Cunningham D, Allum WH, Stenning SP, Thompson JN, Van de Velde CJ, Nicolson M, et al. MAGIC Trial Participants. Perioperative chemotherapy versus surgery alone for resectable gastroesophageal cancer. N Engl J Med. 2006;355:11-20.

9. Boige V, Pignon J, Saint-Aubert B, Lasser P, Conroy T, Bouche O. Final results of a randomized trial comparing preoperative 5-fluorouracil (F)/cisplatin (P) to surgery alone in adenocarcinoma of stomach and lower esophagus (ASLE): FNLCC ACCORD07-FFCD 9703 trial. J Clin Oncol. 2007;25:4510.

10. Brenner B, Shah MA, Karpeh MS, Gonen M, Brennan MF, Coit DG, et al. A phase II trial of neoadjuvant cisplatin-fluorouracil followed by postoperative intraperitoneal floxuridine-leucovorin in patients with locally advanced gastric cancer. Ann Oncol. 2006;17:1404-11.

11. Yoshikawa T, Sasako M, Yamamoto S, Sano T, Imamura H, Fujitani K, et al. Phase II study of neoadjuvant chemotherapy and extended surgery for locally advanced gastric cancer. Br J Surg. 2009;96:1015-22.

12. Sakuramoto S, Sasako M, Yamaguchi T, Kinoshita T, Fujii M, Nashimoto A, et al. ACTS-GC Group. Adjuvant chemotherapy for gastric cancer with S-1, an oral fluoropyrimidine. N Engl J Med. 2007;357:1810-20.

13. National Cancer Institute. Common Toxicity Criteria version 2.0 (CTC). http://ctep.cancer.gov/protocolDevelopment/electronic_ applications/ctc.htm [accessed December, 2010].
14. Japanese Gastric Cancer Association. Japanese classification of gastric carcinoma, 2nd edition. Gastric Cancer. 1998;1:10-24.

15. Fukuya T, Honda H, Hayashi T, Kaneko K, Tateshi Y, Ro T, et al. Lymph-node metastases: efficacy for detection with helical CT in patients with gastric cancer. Radiology. 1995;197:705-11.

16. D'Elia F, Zingarelli A, Palli D, Grani M. Hydro-dynamic CT preoperative staging of gastric cancer: correlation with pathological findings. A prospective study of 107 cases. Eur Radiol. 2000;10:1877-85.

17. Biondi A, Persiani R, Cananzi F, Zoccali M, Vigorita V, Tufo A, et al. R0 resection in the treatment of gastric cancer: room for improvement. World J Gastroenterol. 2010;16:3358-70.

18. D’Ugo DM, Pende V, Persiani R, Rausei S, Picciocchi A. Laparoscopic staging of gastric cancer: an overview. J Am Coll Surg. 2003;196:965-74.

19. Ozmen MM, Zulfikaroglu B, Ozalp N, Ziraman I, Hengirmen S, Sahin B. Staging laparoscopy for gastric cancer. Surg Laparosc Endosc Percutan Tech. 2003;13:241-4.

20. Blackshaw GR, Barry JD, Edwards P, Allison MC, Thomas GV, Lewis WG. Laparoscopy significantly improves the perceived preoperative stage of gastric cancer. Gastric Cancer. 2003;6: 225-9.

21. Therasse P, Arbuck SG, Eisenhauer EA, Wanders J, Kaplan RS, Rubinstein L, et al. New guidelines to evaluate the response to treatment in solid tumors. European Organization for Research and Treatment of Cancer, National Cancer Institute of the United States, National Cancer Institute of Canada. J Natl Cancer Inst. 2000;92:205-16

22. Fukagawa T, Katai H, Saka M, Morita S, Sasajima Y, Taniguchi $\mathrm{H}$, et al. Significance of lavage cytology in advanced gastric cancer patients. World J Surg. 2010;34:563-8.

23. Korenaga D, Tsujitani S, Haraguchi M, Okamura T, Tamada R, Sugimachi K, et al. Long-term survival in Japanese patients with far advanced carcinoma of the stomach. World J Surg. 1988;12: $236-40$.

24. Kikuchi S, Tsukamoto H, Mieno H, Sato K, Kobayashi N, Shimao $\mathrm{H}$, et al. Results of resection of gastric cancer with distant metastases. Hepatogastroenterology. 1998;45:592-6.

25. Maekawa S, Saku M, Maehara Y, Sadanaga N, Ikejiri K, Anai H, et al. Surgical treatment for advanced gastric cancer. Hepatogastroenterology. 1996;43:178-86.

26. Miyashiro I, Takachi K, Doki Y, Ishikawa O, Ohigashi H, Murata $\mathrm{K}$, et al. When is curative gastrectomy justified for gastric cancer with positive peritoneal lavage cytology but negative macroscopic peritoneal implant? World J Surg. 2005;29:1131-4.

27. Mezhir JJ, Shah MA, Jacks LM, Brennan MF, Coit DG, Strong VE. Positive peritoneal cytology in patients with gastric cancer: natural history and outcome of 291 patients. Ann Surg Oncol. 2010;17:3173-80.

28. Badgwell B, Cormier JN, Krishnan S, Yao J, Staerkel GA, Lupo PJ, et al. Does neoadjuvant treatment for gastric cancer patients with positive peritoneal cytology at staging laparoscopy improve survival? Ann Surg Oncol. 2008;15:2684-91.

29. Lorenzen S, Panzram B, Rosenberg R, Nekarda H, Becker K, Schenk U, et al. Prognostic significance of free peritoneal tumor cells in the peritoneal cavity before and after neoadjuvant chemotherapy in patients with gastric carcinoma undergoing potentially curative resection. Ann Surg Oncol. 2010;17:2733-9.

30. Kodera Y, Ito S, Mochizuki Y, Kondo K, Koshikawa K, Suzuki $\mathrm{N}$, et al. A phase II study of radical surgery followed by postoperative chemotherapy with S-1 for gastric carcinoma with free cancer cells in the peritoneal cavity (CCOG0301 study). Eur J Surg Oncol. 2009;35:1158-63.

31. Satoh S, Hasegawa S, Ozaki N, Okabe H, Watanabe G, Nagayama S, et al. Retrospective analysis of 45 consecutive patients with advanced gastric cancer treated with neoadjuvant 
chemotherapy using an S-1/CDDP combination. Gastric Cancer. 2006;9:129-35.

32. Mori T, Fujiwara Y, Yano M, Tamura S, Yasuda T, Takiguchi S, et al. Prevention of peritoneal metastasis of human gastric cancer cells in nude mice by $\mathrm{S}-1$, a novel oral derivative of 5-fluorouracil. Oncology. 2003;64:176-82.
33. Kodera Y, Ishiyama A, Yoshikawa T, Kinoshita T, Ito S, Yokoyama $\mathrm{H}$, et al. A feasibility study of postoperative chemotherapy with S-1 and cisplatin (CDDP) for gastric carcinoma (CCOG0703). Gastric Cancer. 2010;13:197-203. 